\title{
Patient and otolaryngologist perceptions of telemedicine during COVID-19 pandemic
}

\author{
Jérôme R. Lechien ${ }^{1,2,3}$ (D) Thomas Radulesco ${ }^{1,4} \cdot$ Lea Distinguin $^{1,3} \cdot$ Younes Chekkoury-Idrissi ${ }^{1,3} \cdot$ Marta P. Circiu ${ }^{1,3}$. \\ Fahd EL Afia ${ }^{1,3}$. Justin Michel ${ }^{4}$. Jean-François Papon ${ }^{5} \cdot$ Stephane Hans ${ }^{3}$
}

Received: 6 November 2020 / Accepted: 13 January 2021 / Published online: 1 February 2021

(c) The Author(s), under exclusive licence to Springer-Verlag GmbH, DE part of Springer Nature 2021

\begin{abstract}
Background The rapid spread of the coronavirus disease 2019 and the implementation of quarantine in many European countries led to a swift change in health care delivery. Telemedicine was implemented in many otolaryngological departments to ensure the continuous care. The purpose of this study is to report our experience about telemedicine in 86 patients consulting virtually in our departments.

Methods A total of 86 patients benefited from telemedicine consultation from April to Mai May 2020. Patients and physicians were invited to fulfill a satisfaction survey over the 3 days after the consultation.

Results Patients consulted in the following fields: laryngology, voice and swallowing $(N=15 ; 17.4 \%)$, head and neck or plastic surgery $(N=34 ; 39.5 \%)$, rhinology $(N=31 ; 36.1 \%)$ and otology $(N=6 ; 7.0 \%)$. Practitioners estimated that the clinical examination would not have changed the consultation issue in $73.2 \%$ of cases. The realization of delayed clinical examination was rapidly necessary in $9.3 \%$ of cases and useless in $33.7 \%$ of cases. Five percent of patients estimated that the consultation did not bring reliable conclusion. Although the majority of patient $(87.7 \%)$ would recommend telemedicine consultation to friend/family in the context of pandemic, only $44.6 \%$ would accept to replace office- consultation by telemedicine consultation outside the pandemic.

Conclusion Telemedicine appears to be an interesting alternative approach in situation of pandemic and lock-down. Because the patient motivation to further participate to telemedicine appears to be conditioned by the context, efforts are still required to understand the patient perception, satisfaction and fears in view of future implementation outside pandemic.
\end{abstract}

Keywords Telemedicine $\cdot$ Telehealth $\cdot$ Care $\cdot$ Video medicine $\cdot$ COVID-19 $\cdot$ Otolaryngology $\cdot$ Benefit $\cdot$ Perceptions

\section{Introduction}

The worldwide spread of coronavirus disease 2019 (COVID19) has forced many governments to impose quarantine, limiting the moves of patients. In this context, telemedicine consultation was implemented for patients who needed to consult otolaryngologist to limit travel, the related virus

Jérôme R. Lechien and Thomas Radulesco have equally contributed to this work and should be regarded as joint first authors.

Jean-François Papon and Stephane Hans have equally contributed to this word and should be regarded as joint senior authors.

Jérôme R. Lechien

jerome.lechien@umons.ac.be

Extended author information available on the last page of the article propagation and to protect caregivers. The stakes were high, especially in Europe where telemedicine is not common [1]. In this study, we briefly presented the experience of three French University departments of otolaryngology-head and neck surgery.

\section{Methods}

\section{Participants, setting}

From April to Mai 2020, data were collected from patients who had video-based telemedicine general visits (not COVID-19 consultation) or call-telemedicine with an experienced otolaryngologist (senior and junior staff, exclusion of resident) from three University Hospitals. Any patient who asked a consultation was included, irrespective to the 
type of consultation (first contact, follow-up, etc.). The video-based telemedicine was implemented to replace scheduled consultations canceled under the national lockdown. Teleconsultation was implemented through Doctolib ${ }^{\circledR}$ Pro system (Doctolib, Paris, France) and a webcam was installed in the computer of the otolaryngologist. Patients used the computer or the camera of the smartphone for the consultation. The local IRB was obtained for the study (IDRCB: 2020-A00832-37).

\section{Teleconsultation system and surveys}

Patients and physicians were invited to fulfill a satisfaction survey over the 3 days after the consultation. Patients were contacted by the secretaries of the department and patients agreed to participate to the video-based consultation and the study. A few minutes before the appointment, patient waited in a virtual waiting room and otolaryngologist was informed that the patient was ready for the consultation. Over the hours post-appointment, patient received a link to complete the survey.

Both patients and practitioners were invited to provide their perception of the telemedicine approach through standardized and structured questionnaires (Online Appendix 1). The survey of patient included the following outcomes: age, gender, waiting time before the consultation, duration of the consultation, saved transport time, distance between homehospital, fear regarding the technical aspect of the virtual consultation, quality of the video (VAS, 1-100), practitioner understanding regarding the main complaint, thought about the usefulness of clinical examination, understanding about the practitioner explanation (consultation conclusion), need to make additional examination, efficacy of the practitioner without examination, desire to have further video-based consultation and overall satisfaction (VAS, 1-100). At the end of the consultation, otolaryngologist had to decide if the patient had to be convened for an office consultation and the realization of clinical examination. The following outcomes included the otolaryngologist survey: waiting time before the consultation, consultation duration, quality of the video (VAS, 1-100), type of consultation (first, follow-up, result announcement, pre-surgery), chief complaint, field (otology, rhinology, head and neck, laryngology), self-perceived usefulness (VAS, 1-100), impact of the clinical examination on the patient management, need to re-call patient for examination and the related delay, type of next appointment and overall satisfaction (VAS, 1-100).

\section{Statistical analysis}

Statistical analyses were performed using the Statistical Package for the Social Sciences for Windows (SPSS version 22.0; IBM Corp, Armonk, NY, USA). Depending on the data, outcome differences between patient groups were evaluated using Mann-Whitney test, Kruskal-Wallis test or $\chi^{2}$ test. Multivariate analysis was used to investigate the relationship between outcomes.

\section{Results}

A total of 86 patients were included. There were 39 females and 47 males. The mean age of patients was $49.1 \pm 18.5$ years old. Patients consulted in the following fields: laryngology, voice and swallowing $(N=15 ; 17.4 \%)$, head and neck or plastic surgery $(N=34 ; 39.5 \%)$, rhinology $(N=31 ; 36.1 \%)$ and otology $(N=6 ; 7.0 \%)$. The following types of consultation were included: first contact/appointment $(N=26 ; 30.2 \%)$, medical or surgical follow-up $(\mathrm{N}=56$; $65.1 \%)$, histological results $(N=2 ; 2.3 \%)$ and preoperative consultation $(N=2 ; 2.3 \%)$.

The consultation outcomes and perceptions of patients and practitioners were available in Tables 1 and 2, respectively. The mean duration of consultation was $13.1 \mathrm{~min}$, which matched with the patient perception (14.3 min). The video quality was evaluated at $62.9 \pm 33.2$ for patients, while practitioners assessed the quality at $77.0 \pm 28.2$. The quality of video was better perceived by physicians compared to patients $(p=0.006)$.

Practitioners estimated that the clinical examination would not have changed the consultation issue in $73.2 \%$ of cases. The realization of delayed clinical examination was rapidly necessary in $9.3 \%$ of cases and useless in $33.7 \%$ of cases (Table 2). The overall satisfaction of practitioners was high, especially in rhinology area $(p=0.041)$ and for the follow-up consultation $(p=0.030)$.

A few patients had technical apprehension. According to the patient perception, the clinical examination was required in $22.1 \%$ of cases, while $5 \%$ estimated that the consultation did not bring reliable conclusion. There were 17 patients (26.7\%) who believed that an office consultation would be better although the pandemic context but only $4.6 \%$ would not recommend this kind of consultation. Outside pandemic, $44.6 \%$ of patients would accept to consult again through telemedicine approach, while $13.9 \%$ did not know. As for physicians, the overall satisfaction of patients was adequate. There was no gender impact on the findings. Patients who had to have additional examination had estimated more frequently that the in-office clinical examination would change the issue of the consultation $(p=0.009)$. There was no significant association between the overall satisfaction of patients and practitioners. 
Table 1 Patient perception outcomes

\begin{tabular}{|c|c|c|}
\hline Patient outcomes & Units & Results \\
\hline $\mathrm{F} / \mathrm{M}$ ratio & $\mathrm{N}$ & $39 / 47$ \\
\hline Consultation features & & $\mathrm{m} \pm \mathrm{SD}$ \\
\hline Waiting time & $\min$ & $8.6 \pm 24.6$ \\
\hline Consultation duration & $\min$ & $14.3 \pm 7.2$ \\
\hline Quality of video & $0-100$ & $62.9 \pm 33.2$ \\
\hline Saved time (ride) & $\min$ & $50.0 \pm 37.5$ \\
\hline Distance home-hospital & $\mathrm{km}$ & $32.7 \pm 50.4$ \\
\hline Patient perception & & $N(\%)$ \\
\hline Technical apprehension & & $16(18.6)$ \\
\hline Clinical examination was necessary & & $19(22.1)$ \\
\hline No issue at the end of the consultation & & $4(5)$ \\
\hline \multicolumn{3}{|c|}{ Office consultation would be better (context) } \\
\hline Yes & & $17(26.7)$ \\
\hline No & & $37(57.0)$ \\
\hline Don’t know & & $11(16.3)$ \\
\hline \multicolumn{3}{|c|}{ Recommend this type of consultation (context) } \\
\hline Yes & & $75(87.7)$ \\
\hline No & & $4(4.6)$ \\
\hline Don’t know & & $7(7.7)$ \\
\hline \multicolumn{3}{|l|}{$\begin{array}{l}\text { I won't do TM consultation again (outside } \\
\text { pandemic) }\end{array}$} \\
\hline Yes & & $38(44.6)$ \\
\hline No & & $35(41.5)$ \\
\hline Don’t know & & $12(13.9)$ \\
\hline \multicolumn{3}{|l|}{ Consultation duration satisfaction } \\
\hline Adequate & & $75(87.2)$ \\
\hline Not adequate & & $11(12.8)$ \\
\hline Overall Satisfaction & $0-100$ & $80.3 \pm 24.5$ \\
\hline
\end{tabular}

$T M$ telemedicine

\section{Discussion}

In Europe, the development of telemedicine raises fears and may meet resistance from many physicians $[1,2]$. The pandemic was an opportunity to develop telemedicine consultation and to assess the acceptation by patients and otolaryngologists [3, 4]. As reported in the present study and in the otolaryngological literature, the primary benefits of telemedicine in a context of pandemic were accessibility to healthcare, to reduce virus spread and time savings. The high satisfaction rates from both patients and otolaryngologists corroborate recent data from the same U.S. study, where authors reported that in a context of pandemic, most patients expressed a willingness to participate in future remote visits [5]. Moreover, regardless of pandemic, our experience shows that telemedicine may be useful to perform rapid triage of otolaryngological indications requiring clinical or additional examination. Telemedicine may be also useful for followup of chronic diseases such as chronic rhinosinusitis [6].
Table 2 Practitioner perception outcomes

\begin{tabular}{lll}
\hline Practitioner outcomes & Units & Results \\
\hline Consultation features & & $\mathrm{m} \pm \mathrm{SD}$ \\
Waiting time & min & $6.4 \pm 8.5$ \\
Consultation duration (real) & min & $13.1 \pm 4.9$ \\
Quality of video & $0-100$ & $77.0 \pm 28.2$ \\
Types of consultation & & $\mathrm{N}(\%)$ \\
First consultation & & $27(31.4)$ \\
Surgical/medical follow-up & & $56(65.1)$ \\
Result announcement & & $2(2.3)$ \\
Pre-surgery & & $1(1.2)$ \\
Subspecialty & & \\
Laryngology & & $15(17.4)$ \\
Head and Neck & & $34(39.5)$ \\
Rhinology & & $31(36.1)$ \\
Otology & & $6(7.0)$ \\
Practitioner perception & & $\mathrm{m} \pm \mathrm{SD}$ \\
Helpful for patient & & $82.8 \pm 25.1$ \\
Decisive examination requirement & & $\mathrm{N}(\%)$ \\
No & & $63(73.2)$ \\
Yes & & $5(5.8)$ \\
Don't know & & $18(21.0)$ \\
Requirement of delayed examination & & \\
No & & $29(33.7)$ \\
Yes & & $49(57.0)$ \\
Quickly & & $8.3 \pm 2.3 \pm 2.3 \pm 2.5$ \\
Satisfaction & & \\
Overall & & \\
Laryngology & & \\
Head and Neck & & \\
Rhinology & & \\
\hline
\end{tabular}

Interestingly, only a few numbers of patients really required clinical examination during the consultation. In the same vein, Hussaini et al. recently reported that telemedicine may be useful as triage of otolaryngologic consults, which may minimize infectious spread and protect clinicians while preserving high-quality patient care [7]. The primary limitation of telemedicine remains the lack of examination, which is particularly perceived as an important limitation by patients, impacting the perception of the issue of the consultation.

In the present study, we do not have enough data to identify the situations where the telemedicine is limited and has to be avoided. However, some common reasons for consultation may be hypothetically identified. According to our experience, we may classify them into two main categories. The first category includes the clinical situations where the otolaryngologist has to make an intervention, i.e., removal of foreign body, nasal packing, removal of otological or nasal packing, voice prosthesis change, etc. This category may include the majority of urgent situations or some short-term 
postoperative appointments where a 'device' has to be removed or changed. To this first category, we may add the wound dressing follow-up. The second category consists of situations where the clinical examination is mandated, which may include the clinical evaluation of the patient anatomy before a surgery (transoral laser or robotic surgery) or the detection of upper aerodigestive tract lesion or infection. Studies with a large number of patients are needed to identify the situations where the telemedicine is useful or inappropriate.

This study reports preliminary results of telemedicine consultation in three University European centers. As preliminary report, the primary weakness is the low number of patients. However, regarding the current second wave of contaminations in Europe, these data may encourage the development of such approach in centers of the countries where there is a second lock-down period. Moreover, the majority of patient consultations in the present study consisted of follow-up consultation and only $30.2 \%$ of consultations were first contact. In that way, it is possible that the good outcomes found in the study results depended on the type of consultation. Thus, future studies are needed to assess the impact of the type of consultation on patient and physician satisfactions.

\section{Conclusion}

Telemedicine appears to be an interesting alternative approach in situation of pandemic and lock-down. However, future studies with a large number of patients are needed to identify the situations where telemedicine is useful or inappropriate. Because the patient motivation to further participate to telemedicine appears to be conditioned by the context, efforts are still required to understand the patient perception, satisfaction and fears in view of future implementation outside pandemic.

Supplementary Information The online version contains supplementary material available at https://doi.org/10.1007/s00405-021-06624-9.

\section{Compliance with ethical standards}

Conflicts of interest The authors have no conflicts of interest.
Ethical approval All procedures performed in studies involving human participants were in accordance with the ethical standards of the institutional and/or national research committee and with the 1964 Helsinki declaration and its later amendments or comparable ethical standards.

Informed consent Informed consent was obtained from patients enrolled in the study.

Research involving human participants and/or animals IRB approved the study protocol (CHUB2020-12).

\section{References}

1. Kayyali R, Hesso I, Mahdi A, Hamzat O, Adu A, Nabhani GS (2017) Telehealth: misconceptions and experiences of healthcare professionals in England. Int J Pharm Pract 25(3):203-209. https ://doi.org/10.1111/ijpp.12340

2. Taylor P (1998) A survey of research in telemedicine. 2: Telemedicine services. J Telemed Telecare. 4(2):63-71. https://doi. org/10.1258/1357633981931948

3. Zughni LA, Gillespie AI, Hatcher JL, Rubin AD, Giliberto JP (2020) Telemedicine and the interdisciplinary clinic model: during the COVID-19 pandemic and beyond. Otolaryngol Head Neck Surg 163(4):673-675. https://doi.org/10.1177/019459982093216 7

4. Shipchandler TZ, Nesemeier BR, Parker NP, Vernon D, Campiti VJ, Anthony BP, Alwani MM, Illing EA, Ting JY (2020) Telehealth opportunities for the otolaryngologist: a silver lining during the COVID-19 pandemic. Otolaryngol Head Neck Surg 163(1):112-113. https://doi.org/10.1177/0194599820929641

5. Triantafillou V, Layfield E, Prasad A, Deng J, Shanti RM, Newman JG, Rajasekaran K (2020) Patient perceptions of head and neck ambulatory telemedicine visits: a qualitative study. Otolaryngol Head Neck Surg. 19:23. https://doi.org/10.1177/0194599820 943523

6. Zhou AS, Prince AA, Maxfield AZ, Corrales CE, Shin JJ (2020) The Sinonasal outcome test-22 or european position paper: which is more indicative of imaging results? Otolaryngol Head Neck Surg. https://doi.org/10.1177/0194599820953834

7. Hussaini AS, Clark CM, Patel AA, Russo ME, Chia SH, Davidson BJ, Malekzadeh S (2020) Management of adult inpatient otolaryngologic consultations during the COVID-19 pandemic: a proposed tier-based triage system. Otolaryngol Head Neck Surg 163(2):330-334. https://doi.org/10.1177/0194599820931011

Publisher's Note Springer Nature remains neutral with regard to jurisdictional claims in published maps and institutional affiliations.

\section{Authors and Affiliations}

\section{Jérôme R. Lechien ${ }^{1,2,3}$ (D) . Thomas Radulesco ${ }^{1,4} \cdot$ Lea Distinguin $^{1,3} \cdot$ Younes Chekkoury-Idrissi ${ }^{1,3} \cdot$ Marta P. Circiu ${ }^{1,3}$. Fahd EL Afia ${ }^{1,3}$. Justin Michel ${ }^{4}$. Jean-François Papon ${ }^{5} \cdot$ Stephane Hans ${ }^{3}$}

1 COVID-19 task force of the Young-Otolaryngologists of the International Federations of Oto-rhino-laryngological Societies (YO-IFOS), Paris, France
2 Department of Human Anatomy and Experimental Oncology, School of Medicine, Research Institute for Health Sciences and Technology, University of Mons, Mons, Belgium 
3 Department of Otolaryngology-Head \& Neck Surgery, Foch Hospital, School of Medicine, UFR Simone Veil,

Université Versailles Saint-Quentin-en-Yvelines (Paris Saclay University), Paris, France

4 Department of Otorhinolaryngology-Head \& Neck Surgery, AP-HM, La Conception Hospital, Marseille, France
5 Department of Otorhinolaryngology-Head \& Neck Surgery, Le Kremlin-Bicêtre Hospital, APHP, Paris Saclay University, Paris, France 